\title{
Extended Overview of the Living Labs for Information Retrieval Evaluation (LL4IR) CLEF Lab 2015
}

\author{
Anne Schuth ${ }^{1}$, Krisztian Balog ${ }^{2}$, and Liadh Kelly ${ }^{3}$ \\ 1 University of Amsterdam, The Netherlands \\ 2 University of Stavanger, Stavanger, Norway \\ 3 ADAPT Centre, Trinity College, Dublin, Ireland \\ anne.schuth@uva.nl, krisztian.balog@uis.no, liadh.kelly@tcd.ie
}

\begin{abstract}
In this extended overview paper we discuss the first Living Labs for Information Retrieval Evaluation (LL4IR) lab which was held at CLEF 2015. The idea with living labs is to provide a benchmarking platform for researchers to evaluate their ranking systems in a live setting with real users in their natural task environments. LL4IR represents the first attempt to offer such experimental platform to the IR research community in the form of a community challenge. For this first edition of the challenge we focused on two specific use-cases: product search and web search. Ranking systems submitted by participants were experimentally compared using interleaved comparisons to the production system from the corresponding use-case. In this paper we describe how these experiments were performed, what the resulting outcomes are, and provide a detailed analysis of the use-cases and a discussion of ideas and opportunities for future development.
\end{abstract}

Keywords: Information retrieval evaluation, living labs, product search, web search.

\section{Introduction}

Evaluation is a central aspect of information retrieval (IR) research. In the past few years, a new evaluation paradigm known as living labs has been proposed, where the idea is to perform experiments in situ, with real users doing real tasks using real-world applications [13]. This type of evaluation, however, has so far been available only to (large) industrial research labs [16, 25]. Our main goal with the Living Labs for IR Evaluation (LL4IR) CLEF Lab is to provide a benchmarking platform for researchers to evaluate their ranking systems in a live setting with real users in their natural task environments. The lab acts as a proxy between commercial organizations (live environments) and lab participants (experimental systems), facilitates data exchange, and makes comparison between the participating systems. This initiative is a first of its kind for IR.

The first edition of the lab focuses on two use-cases and one specific notion of what a living lab is (with a view to expanding to other use-cases and other interpretations of living labs in subsequent years). Use-cases for the first lab are: product search (on an e-commerce site) and web search (through a large commercial web search engine). For each of the two use-cases, challenge participants receive a set of frequent queries along 
with candidate documents for each of these queries. For the product search use-case, each document represents a product with attributes. This use-case also has actual query strings and historical click log data. The other use-case, web-search, only provides feature vectors. There is no content for documents nor query strings. The web-search usecase represents a typical learning to rank setting [17].

Given the queries and documents, participants produce rankings for each query and upload these rankings through an API. These rankings are evaluated online, on real users, using an interleaved comparison method [20]. Interleaving performs a pairwise comparison by presenting each user with a result list that is a combination of the rankings from the participant and the production systems. The results of these evaluations (interleaving outcomes) are made available to the participants, again, through an API. During the training period, participants are free to update their rankings using this feedback information. It is not allowed to make changes to the rankings during the test phase. Using a shared API but considering two different application scenarios allows us to study how well online evaluation techniques generalize across domains.

The official CLEF evaluation round took place between May 1 and May 15, 2015. The positive feedback and growing interest from participants motivated us to organize a subsequent second evaluation round, from Jun 1 to Jun 15, 2015. We will refer to these as Round \#1 and Round \#2 of the LL4IR Challenge, respectively. It is planned that further challenge rounds will be organized continuously ( 2 weeks every month) so that participants do not have to wait for an annual event to test their developments. ${ }^{4}$

In the next section we provide a brief background on online evaluation. In Section 3 we describe the LL4IR Challenge operation and give extensive details on our architecture. We then describe each of the two use-cases of the first edition of the lab in turn in Sections 4 and 5, and provide details and analysis of the submissions received. Finally, in Section 6, we conclude the paper.

\section{Background}

In this section we firstly introduce related ideas regarding living labs in general. We then provide background on evaluation, and in particular on online evaluation.

\subsection{Living Labs}

Ideas for living labs for IR instances have been proposed over the last few years [2, 14]. In particular, Azzopardi and Balog [2] describe in great detail what is need to go from a more traditional IR setting to a full fledged living lab scenario. Their setup bears many similarities to our setup, but differences can be found in that we restrict ourself to head queries and that we view our living labs infrastructure purely as a proxy. Kelly et al. [14] focused more on a living lab setting as a solution to the evaluation of personal search. We do not have this focus.

The need for more realistic evaluation, involving real users, was reiterated at several recent IR workshops [1, 3, 12]. The Living Labs for Information Retrieval Evaluation

\footnotetext{
${ }^{4}$ See http://living-labs. net/challenge/for details on the continuous challenge.
} 
(LL'13) workshop at CIKM two years ago was a first attempt to bring people, both from academia and industry, together to discuss challenges and to formulate practical next steps. The workshop was successful in identifying and documenting possible further directions [3]. Our main goals now are to continue our community building efforts around living labs for IR and to pursue the directions set out at LL'13. The first Living Labs for Information Retrieval Evaluation Challenge (LL4IR) lab at CLEF $2015^{5}$ contributes towards this goal. Having a community benchmarking platform with shared tasks is a key catalyst in enabling people to make progress in this area. Towards developing the challenge for the CLEF lab, we dedicated a separate event in 2014, the Living Labs Challenge (LLC) workshop ${ }^{6}$, to the testing and further development of our challenge platform.

Our initiative is a first of its kind for IR. CLEF Newsreel [6] ${ }^{7}$ is a similar initiative, but for a different problem domain: news recommendation. By contrast we are focusing on the very different space of information retrieval, which contains its own unique use-cases, approaches, challenges, and researchers. Major differences between the labs include the presence of a query and, importantly, that our API lifts the real time processing requirements on the part of participants, lowering the participation threshold significantly.

\subsection{Evaluation}

Evaluation has always played an important role in IR research. Traditionally, evaluation was performed offline following the Cranfield paradigm, using static test collections [21]. This paradigm ensures reproducibility, however, real user satisfaction is not always accurately reflected by this way of evaluating systems [27, 28]. An explanation for the discrepancy is that the relevance judgments used in these static test collections come from expert annotators, not from the users themselves. This is one of the major reasons for performing online evaluation: evaluation with users in the loop. It is also one of the main reasons for initiating our living labs for IR initiative. Initially, user interactions with IR systems were observed and metrics such as the click through rate and time to click were computed. These metrics then served as a means of comparing two systems. However, more recently, interleaved comparisons were shown to be two orders of magnitude more sensitive than other ways of performing online evaluation such as A/B testing [7, 25]. This means that far fewer query impressions are required to make informed decisions on which ranker is better. In our living labs, we use interleaved comparisons as the main evaluation metric. Below we provide details on how interleaving works.

Interleaved comparisons Many interleaving approaches have been proposed over the past few years (for instance [9, 11, 19, 20, 23, 24]). By far the most frequently used interleaving algorithm to date is Team Draft Interleaving (TDI) [20] which is also what is used in our living labs. Given a user query $q$, TDI produces an interleaved result list

\footnotetext{
${ }^{5}$ See http://living-labs.net/clef-lab.

${ }^{6}$ See http: //living-labs.net/llc.

${ }^{7}$ See http: //www.clef-newsreel.org.
} 
as follows. The algorithm takes as input two rankings. One ranking from the participant $r^{\prime}=\left(a_{1}, a_{2}, \ldots\right)$ and one from the production system $r=\left(b_{1}, b_{2}, \ldots\right)$. The goal is to produce a combined, interleaved ranking $L=\left(a_{1}, b_{2}, \ldots\right)$. This is done analogue to how sports teams may be constructed in a friendly sports match. The two team captains take turns picking players. They can pick available documents (players) from the top of the rankings $r^{\prime}$ and $r$, these top ranked document are deemed to be the best documents. Documents can only be picked once (even if they are listed in both $r$ and $r^{\prime}$ ). And the order in which the documents are picked determines ranking $L$. In each round, the team captains flip a coin to determine who goes first. The algorithm remembers which team each document belongs to. If a document receives a click from a user, credit is assigned to the team the document belongs to. The team (participant or production system) with most credit wins the interleaved comparison. This process is repeated for each query. For more details see the original paper describing TDI by Radlinski et al. [20] and a large scale comparison of interleaving methods by Chapelle et al. [7].

\section{The Living Labs Architecture}

Our livings labs evaluation methodology heavily depends on a novel architecture which we describe in this section. We start with an overview, we then introduce the organization of our challenge, and we finish with implementation details.

\subsection{Overview}

For each of the use-cases, described in Sections 4 and 5, challenge participants take part in a live evaluation process. For this they use a set of frequent queries as training queries and a separate set of frequent queries as test queries. Candidate documents are provided for each query along with historical information associated with the queries. When participants produce their rankings for each query, they upload these through the provided LL4IR API. The commercial provider then interleaves a given participant's ranked list with their own ranking, and presents the user with the interleaved result list. Participants take turns in having their ranked list interleaved with the commercial provider's ranked list. This process of interleaving a single experimental system with the production system at a time is orchestrated by the LL4IR API, such that each participant gets about the same number of impressions. The actions performed by the users of the commercial provider's system are then made available to the challenge participant (whose ranking was shown) through the API; i.e., the interleaved ranking, resulting clicks, and (aggregated) interleaving outcomes.

Figure 1 shows the Living Labs architecture and how the participant interacts with the use-cases through the LL4IR provided API. As can be seen, frequent queries $(Q)$ with candidate documents for each query $(D \mid Q)$ are sent from a site through the API to the experimental systems of participants. These systems upload their rankings $\left(r^{\prime}\right)$ for each query to the API. When a user of the site issues one of these frequent queries $(q)$, then the site requests a ranking $\left(r^{\prime}\right)$ from the API and presents it interleaved with $r$ to the users. Any interactions $(c)$ of the user with this ranking are sent back to the API. Experimental systems can then obtain these interactions $(c)$ from the API and 


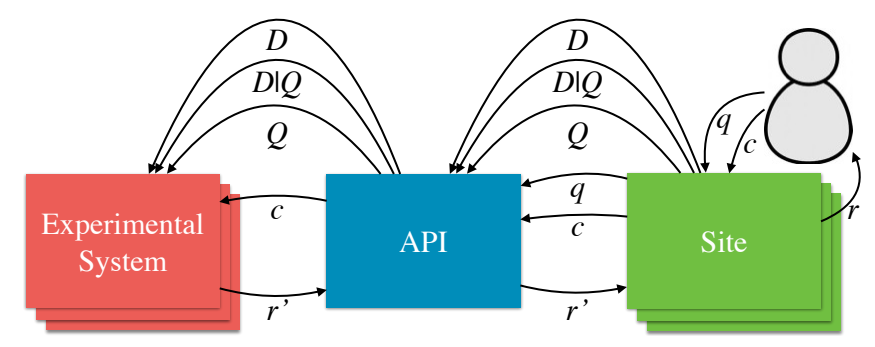

Fig. 1. Schematic representation of interaction with the LL4IR API, taken from [4].

update their ranking $\left(r^{\prime}\right)$ if they wish. We provided participants with example code and guidelines to ease the adaptation to our setup. ${ }^{8}$ Our evaluation methodology is described in more detail in [4].

Head Queries The distribution of search queries typically follows a power law [26], where a relatively small set of head queries are frequently posed by many users and there is a long tail of queries that appear in the logs only a few times (often only once). In our livings labs setting, we focus exclusively on head queries for a number of reasons:

1. this allows us to evaluate experimental search systems on the same set of queries;

2. these queries have a stable volume level, even for mid-sized sites (cf. [4]); and

3. historical click and usage data is available in meaningful quantities.

\subsection{Challenge Organization}

Much like any other (information retrieval or machine learning) evaluation campaign, we split our the data into training and testing parts. Given that we perform online evaluation, it is slightly more involved than simply providing disjoint datasets. We describe our setup here.

Training Phase During the training phase, participants are free to update their rankings using feedback information. This feedback information is made available to them as soon as it arrives at the API. Their rankings can be updated at any time and as often as desired. Both click feedback and aggregated outcomes are made available directly and are updated constantly.

Test Phase In the test phase, challenge participants receive another set of frequent queries as test queries. Again, the associated historical click information as well as candidate results for these queries are made available. After downloading the test queries, participants could only upload their rankings until the test phase started or only once after it started. These rankings are then treated in the same way as training queries. That

${ }^{8}$ http://doc.living-labs.net/en/latest/guide-participant.html 
is, they are interleaved with the commercial providers' rankings for several weeks. As for the training phase, in the test phase each challenge participant is given an approximately equal number of impressions. A major difference is that for the test queries, the click feedback is not made available. Aggregated outcomes are provided only after the test phase ends.

As detailed before, the official CLEF evaluation phase took place between May 1 and May 15, 2015. The positive feedback and growing interest from participants motivated us to organize a subsequent second evaluation round, from Jun 1 to Jun 15, 2015. We will refer to these as Round \#1 and Round \#2 of the LL4IR Challenge, respectively. It is planned that further challenge rounds will be organized continuously (2 weeks every month) so that participants do not have to wait for an annual event to test their developments.

\subsection{Evaluation Metric}

The overall evaluation of challenge participants is based on the final system performance, and additionally on how the systems performed at each query issue. The primary metric used is aggregated interleaving outcomes, and in particular we are interested in the fractions of winning system comparisons.

Interleaved comparisons In Section 2.2 we provided details on how interleaving, and in particular Team Draft Interleaving (TDI) [20], works. TDI is used by both our use-cases. There are two reasons for using interleaved comparisons. Firstly, interleaved comparisons were shown to be two orders of magnitude more sensitive than other ways of performing online evaluation such as A/B testing [7, 25]. Secondly, interleaved comparisons ensure that at least half the ranking shown to users comes from the production system. This reduces the risk of showing bad rankings to users.

Aggregated Outcomes We report the following aggregated interleaving metrics, where Outcome serves as the primary metric for comparing participants rankings. These aggregations are constantly updated for training queries. For the test phase they are only computed after the phase is over.

\#Wins is defined as the number of wins of the participant against the production system, where a win is defined as the experimental system having more clicks on results assigned to it by TDI than clicks on results assigned to the production system;

\#Losses is defined as the number of losses against the production system;

\#Ties is defined as the number of ties with the production system;

\#Impressions is the total number of times when rankings (for any of the test queries) from the participant have been displayed to users of the production system; and

Outcome is defined as the fraction of wins, so \#Wins/(\#Wins+\#Losses).

An Outcome value below the expected outcome (typically 0.5) means that the participant system performed worse than the production system (i.e., overall it has more losses than wins). Significance of outcomes is tested using a two-sided binomial test which uses the expected outcome; p-values are reported. 
Table 1. Overview of API endpoints with a short description. For a full description including examples, please see http://doc.living-labs.net/en/latest/ api-participant.html.

\begin{tabular}{lll}
\hline Method Endpoint & Description \\
\hline GET & /api/participant/query/(key) & $\begin{array}{l}\text { Obtain the query set. Each query is marked } \\
\text { with its type. }\end{array}$ \\
GET & /api/participant/doclist/(key)/(qid) & $\begin{array}{l}\text { Retrieve the document list for a query. This } \\
\text { doclist defines the set documents that are re- } \\
\text { turnable for a query. } \\
\end{array}$ \\
GET & /api/participant/doc/(key)/(docid) & $\begin{array}{l}\text { Retrieve the actual content of a single doc- } \\
\text { ument. }\end{array}$ \\
PUT & /api/participant/run/(key)/(qid) & $\begin{array}{l}\text { Submit a run (ranking) for a specific query. } \\
\text { GET }\end{array} \quad$ /api/participant/feedback/(key)/(qid)/(runid) $\begin{array}{l}\text { Obtain feedback for a query. Only feedback } \\
\text { for runs by the given participant will be re- } \\
\text { turned. }\end{array}$ \\
& $\begin{array}{l}\text { Obtain outcomes. Outcome will be aggre- } \\
\text { gated per site and for test and train queries. }\end{array}$ \\
GET & /api/participant/outcome/(key)/(qid) &
\end{tabular}

Note that using these metrics, we are in theory only able to say something about the relationship between the participant's system and the production system. However, Radlinski et al. [20] show experimentally that it is not unreasonable to assume transitivity. This allows us to also draw conclusions about how systems compare to each other. Ideally, instead of interleaving, we would have used multileaved comparison methods $[23,24]$ which would directly give a ordering over rankers by comparing them all at once for each query. However, multileaved comparisons could potential impact users more than they would be impacted by interleaving. Moreover, multileaved comparison methods are not yet as established as an evaluation method.

\subsection{Implementation}

Our living labs API, as shown in Figure 1 and described in Section 3.1, is a middleman for queries, rankings and clicks. The API talks to both sites (the use-cases) and participants (the researchers). In this paper, we only describe how it interacts with participants. The interaction with sites is described in detail in the documentation. ${ }^{9}$

Our API is implemented as a REST-full service, meaning that communication uses the HTTP protocol and that URLs describe resources. All communication is encoded in JSON. Table 1 lists all the endpoints participants can communicate with. Below we describe them in some more detail.

Queries Participants would typically start by downloading the head queries for each use-case. They can do so using this endpoint:

GET /api/participant/query/(key)

\footnotetext{
${ }^{9}$ For our documentation, see http: // doc. living-labs . net.
} 
The only parameter the participant needs to provide is the key which is their API key. Note that participants will only receive queries for the use-case they signed up for.

Each query is marked with its type. A query can be a "train" or "test" query indicating the phase they belong to, see Section 3.2. The default query type is "train". Queries have an identifier ( $q i d$ ) and, depending on the use-case, an actual query string (qstr). Participants should regularly update their query set. An example output would look like this:

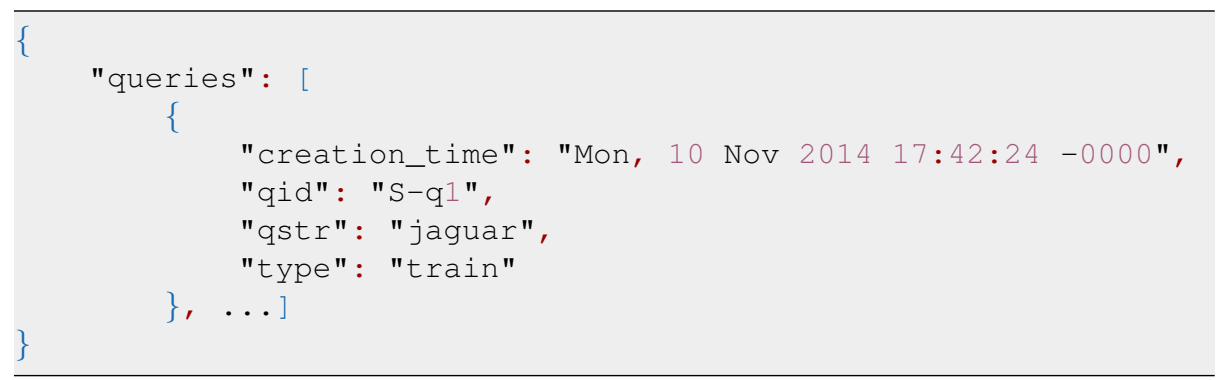

Candidate documents Secondly, for each query, a set of candidate documents is provided. In order to obtain these, this endpoint is used:

GET /api/participant/doclist/(key)/(qid)

The set of documents is provided as a list of document identifiers. This list can change over time as new documents arrive or old documents go stale. See the example output:

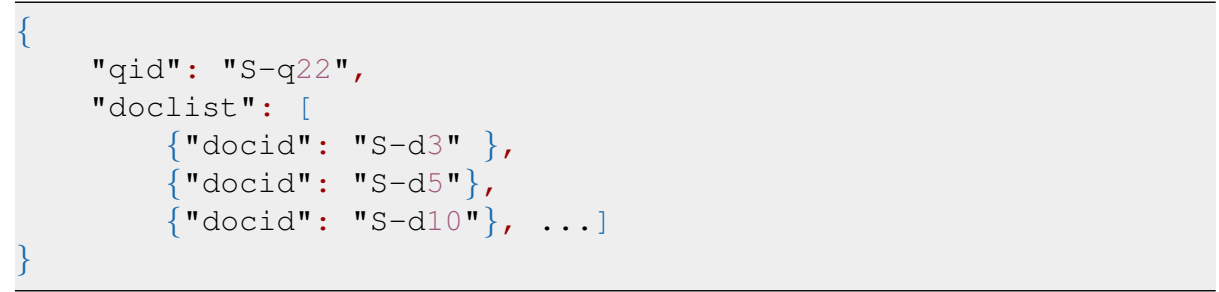

Note that depending on the use-case, relevance signals may also be provided for each document. See Section 5 for details.

Documents details Lastly, participants will need the actual content of documents. The endpoint we provide for this purpose is:

GET /api/participant/doc/(key)/(docid)

An example document would look like this:

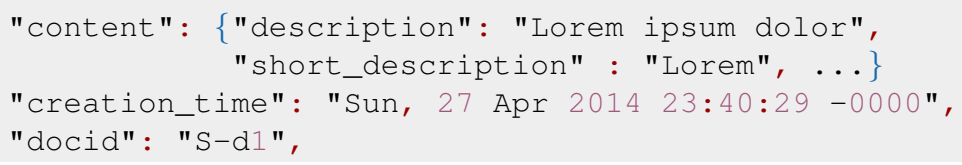




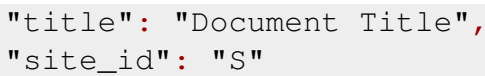

The content of the document can be a simple string, or potentially it can have structure. In which case it would be a JSON object. Note that depending on the use-case, these documents may be empty, again see Section 5 for details.

Run After the participant obtains queries, candidate document sets and the content of documents, runs (rankings) can be prepared. These runs can then be uploaded by the participant to the API using this endpoint:

PUT /api/participant/run/(key)/(qid)

An example run would look like this:

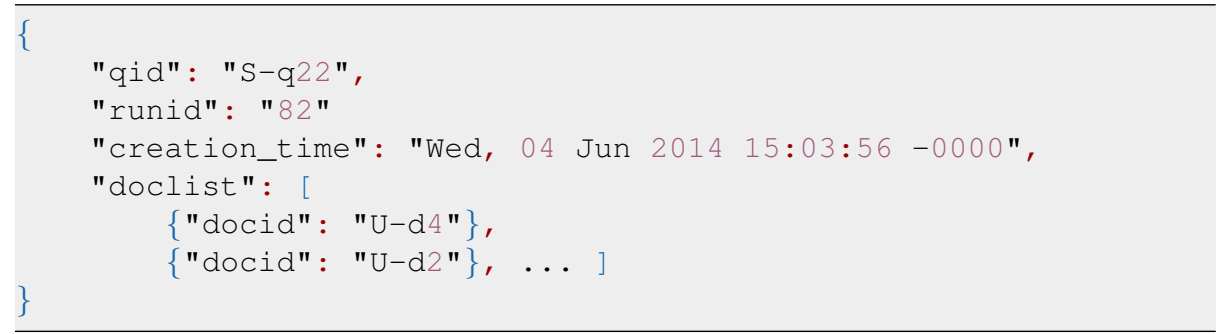

This run provides the query for which it is a ranking ( $q i d$ ) and it provides a runid. Note that the runid is only for the participants own bookkeeping. It could be any string, for instance a timestamp or the version of the ranker.

Once uploaded, the run is displayed to users (interleaved with the commercial provider's results) when the given query is issued. If other participants also uploaded a ranking for this query, the run to show to the given user is sampled uniformly from the available runs.

Note that for test queries (cf., Section 3.2) the runs can only be submitted before a test phase starts. Our API will reject runs for test queries during the testing phase.

Feedback Once runs are submitted and exposed to users, their interactions are logged. The interactions with their own runs can be retrieved by participants using the feedback endpoint:

GET /api/participant/feedback/(key)/(qid)/(runid)

An example of feedback returned by our API would look like this:

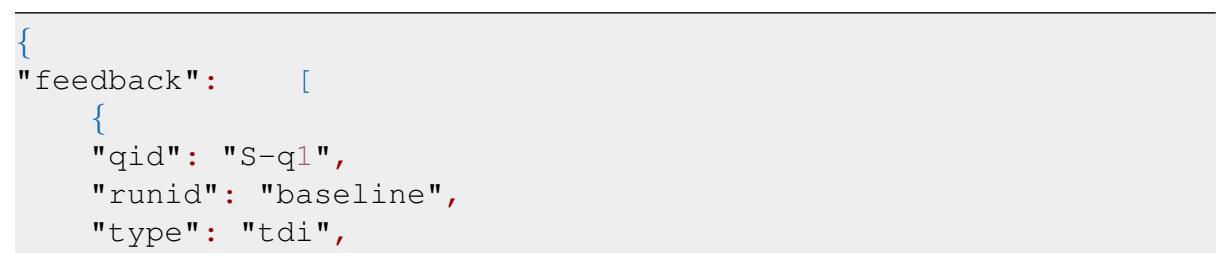




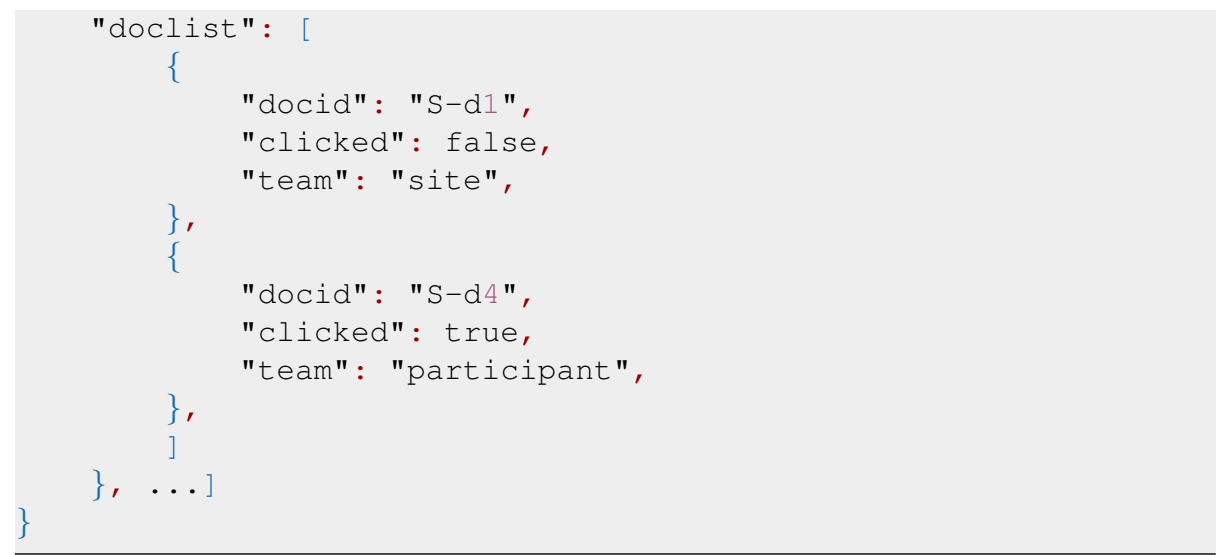

In this feedback, the ranking that was shown to the user is provided for train queries (cf., Section 3.2). The runid is simply the run identifier provided by the participant. The ranking in the above example was a TDI ranking (cf., Section 2.2), an interleaving of the participant's run with the production ranking from the site. The team field shows to which team each document was assigned. The clicked field simply indicates whether the document was clicked. This feedback is per impression, so not aggregated. Note that there can be multiple feedbacks for the same runid. Participants can use the feedback as they see fit, but typically one would compute whether the site or the participant won the interleaved comparisons, see Section 2.2 for details on how to do this.

Outcome Finally, all interactions of users with the runs from participants can be aggregated into outcomes. These aggregated results are provided as an API endpoint at:

GET /api/participant/outcome/(key)/(qid)

An example outcome would look like this:

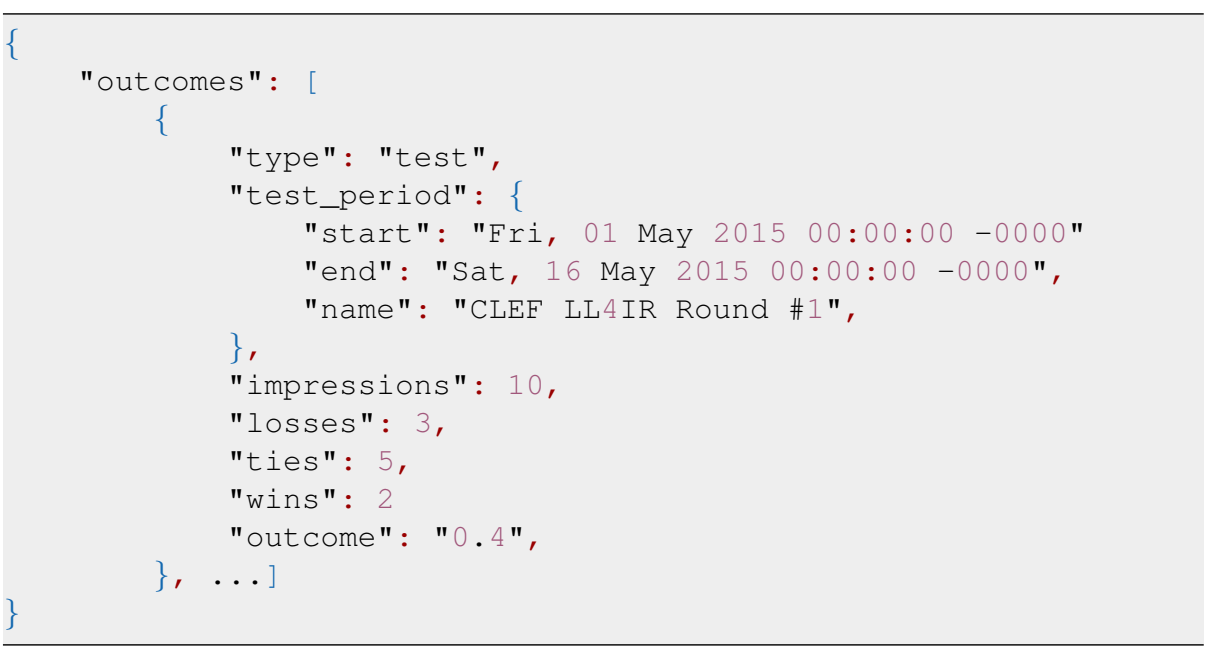


In this example, we see the aggregation of outcomes over 10 impressions of the runs from a single participant for a single query; the participant lost more impressions than it won. The query identifier qid can be omitted in which case the outcome would be aggregated over all queries. Outcomes are split out per train and test phase (cf., Section 3.2). In case the participant participated in multiple test phases with the same query, multiple aggregations are shown.

\section{Use-case 1: Product Search}

\subsection{Task and Data}

The product search use-case is provided by REGIO Játék (REGIO Toy in English), the largest (offline) toy retailer in Hungary with currently over 30 stores. Their webshop ${ }^{10}$ is among the top 5 in Hungary. The company is working on strengthening their online presence; improving the quality of product search in their online store is directed towards this larger goal. An excerpt from the search result page is shown in Figure 2.

As described in Section 3, we distinguish between training and test phases. Queries are sampled from the set of frequent queries; these queries are very short (1.18 terms on average) and have a stable search volume. For each query, a set of candidate products (approximately 50 products per query) and historical click information (click through rate) is made available. For each product a structured representation is supplied (see below). The task then is to rank the provided candidate set.

Product Descriptions For each product a fielded document representation is provided, containing the attributes shown in Table 2. The amount of text available for individual products is limited (and is in Hungarian), but there are structural and semantic annotations, including:

- Organization of products into a two-level deep topical categorization system;

- Toy characters associated with the product (Barbie, Spiderman, Hello Kitty, etc.);

- Brand (Beados, LEGO, Simba, etc.);

- Gender and age recommendations (for many products);

- Queries (and their distribution) that led to the given product.

Candidate Products The candidate set, to be ranked, contains all products that were available in the (recent) past. This comprises all products that are considered by the site's production search engine (in practice: all products that contain any of the query terms in any of their textual fields). One particular challenge for this use-case is that the inventory (as well as the prices) are constantly changing; however, for challenge participants, a single ranking will be used throughout the entire test period of the challenge, without the possibility of updating it. The candidate set therefore also includes products that may not be available at the moment (but might become available again in the future). Participating systems were strongly encouraged to consider all products from the

\footnotetext{
${ }^{10}$ http: //www . regiojatek.hu/
} 


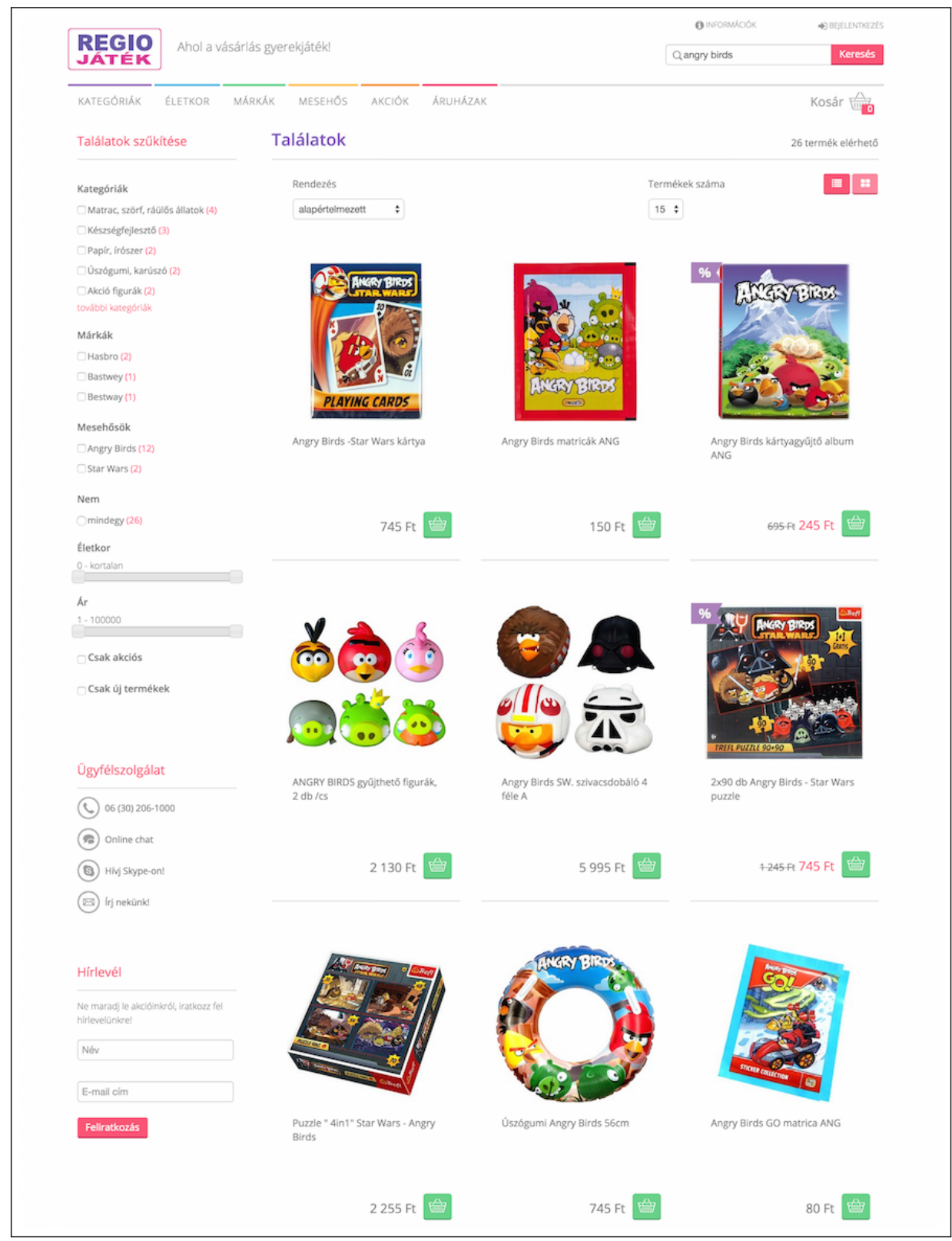

Fig. 2. Screenshot of REGIO, our product search use-case.

provided candidate set. Those that were unavailable at a given point in time were not displayed to users of the REGIO online store. Further, it may happen (and as we show in Section 4.3 it indeed does happen) during the test period that new products arrive; 
Table 2. Fielded document representation of products in the product search use-case.

\begin{tabular}{|c|c|}
\hline Field & Description \\
\hline age_max & Recommended maximum age (may be empty, i.e., 0 ) \\
\hline age_min & Recommended minimum age (may be empty, i.e., 0 ) \\
\hline arrived & $\begin{array}{l}\text { When the product arrived (first became available); only for products that } \\
\text { arrived after 2014-08-28 }\end{array}$ \\
\hline available & Indicates if the product is currently available (1) or not (0) \\
\hline bonus_price & Provided only if the product is on sale; this is the new (sales) price \\
\hline brand & Name of the brand (may be empty) \\
\hline category & Name of the (leaf-level) product category \\
\hline category_id & Unique ID of the (leaf-level) product category \\
\hline characters & List of toy characters associated with the product (may be empty) \\
\hline description & Full textual description of the product (may be empty) \\
\hline main_category & Name of the main (top-level) product category \\
\hline main_category_id & Unique ID of the main (top-level) product category \\
\hline gender & $\begin{array}{l}\text { Gender recommendation. ( } 0 \text { : for both girls and boys (or unclassified); } \\
\text { 1: for boys; } 2 \text { : for girls) }\end{array}$ \\
\hline photos & List of photos about the product \\
\hline price & Normal price \\
\hline product_name & Name of the product \\
\hline queries & $\begin{array}{l}\text { Distribution of (frequent) queries that led to this product (may be } \\
\text { empty) }\end{array}$ \\
\hline short_description & Short textual description of the product (may be empty) \\
\hline
\end{tabular}

experimental systems are not able to include these in their ranking (this is the same for all participants), while the production system might return them. This can potentially affect the number of wins against the production system (to the advantage of the production system), but it will not affect the comparison across experimental systems.

\subsection{Implementation}

While we do not want to get into technical details here, there are three specific issues worth mentioning regarding the interleaving implementation at the site's end. First, search typically involves a pipeline of components: query analysis (including, e.g., spell correction), ranking, filtering (e.g., considering facets), and finally presenting the SERP (with pagination, etc.). It is important that interleaving takes place in the ranking component, before any filtering is applied. Second, the rankings submitted by participants may include products that are currently not available. These need to be removed before the interleaving is performed. Finally, the same user should be presented with the same (interleaved) ranking for a given query throughout her session (in case the query is issued multiple times); this caching mechanism needs to be implemented by the site.

\subsection{Inventory Changes}

One peculiarity of the product search use-case is that the inventory is subject to frequent changes. Not all products that are part of the candidate set are available at all 


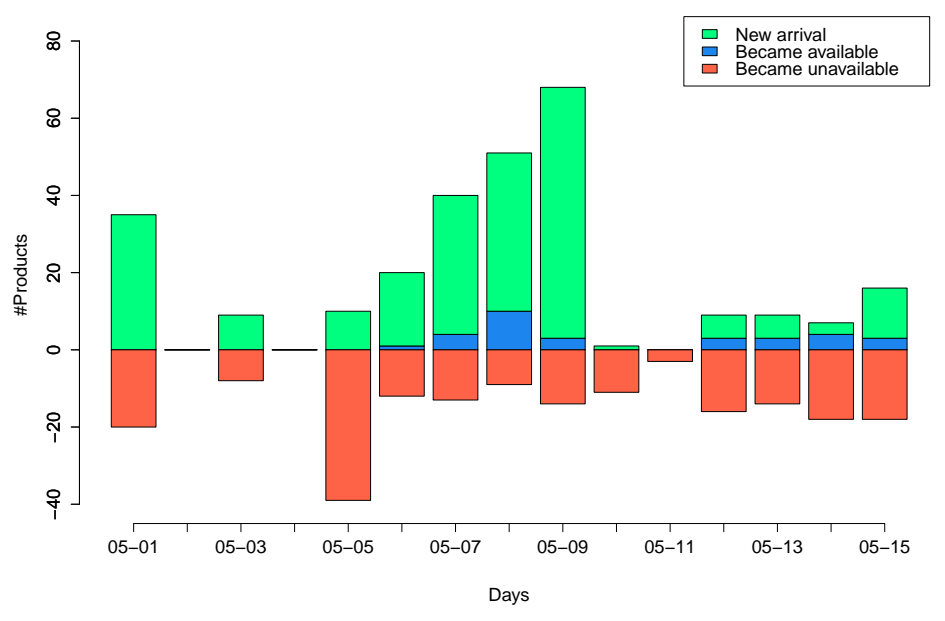

Fig. 3. Inventory changes during Round \#1 of the challenge.

times. Also, new products may arrive over time that are not available to experimental systems but do get returned by the production system (and hence get clicked by users). We further note that new arrivals are displayed distinctively in the webshop, which may also introduce a bias. Figure 3 displays the number of new arrivals (in green), and the products that became available (blue) or unavailable (red) from the day before. Only products that are candidates for any of the queries (either training or test) are considered. This figure shows absolute numbers. It is apparent that changes do occur, and in particular a great number of new products arrive. (This is actually the least desired type of change, as there is no easy way of dealing with it in our current platform.) An even more revealing statistic would be to measure the ratio of products that were unavailable at a given day, compared to all candidate products that were ever available during the test phase. This is shown in Figure 4. Note that unavailability ratio is specific to a given ranking; the reported numbers are computed for the organizers' baseline. To keep things simple, we use a single value, the average unavailability ratio of all submitted rankings, which is $44 \%$.

If all products were available, the expected probability of winning an interleaved comparison (assuming a randomly clicking user) would be 0.5 . However, on average, $44 \%$ of the products were actually unavailable. During Round \#1, these products were only ever present in the participants ranking (the site's ranking never considered them). And, only after interleaving were these products removed from the resulting interleaved list. We note that this is undesired behavior, as they should have been filtered out before interleaving. The necessary adjustments were made to the implementation for Round \#2 of the challenge. As for interpreting the Round \#1 results, this means that the chances for products from the participants ranking to be clicked were reduced. We believe that 


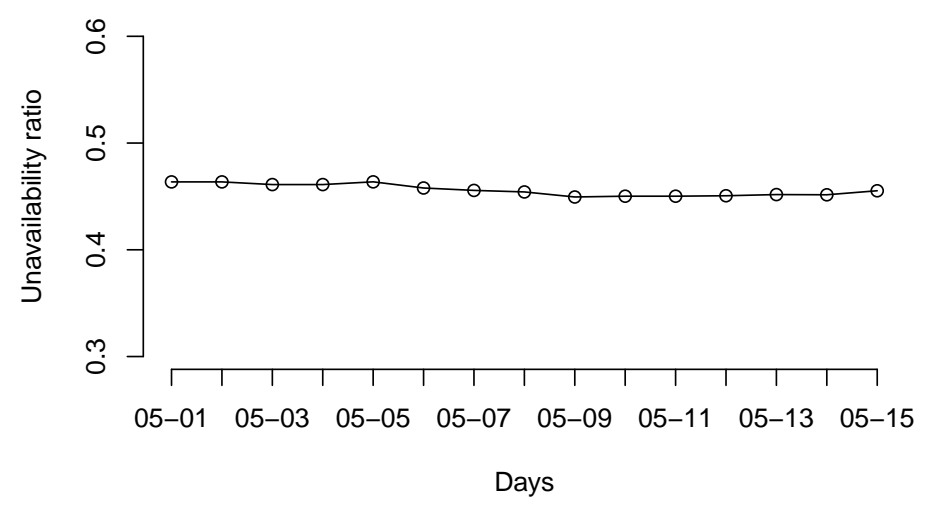

Fig. 4. Unavailability ratio for the organizers' baseline during Round \#1 of the challenge.

this in turn reduces the expected probability to win to:

$$
P(\text { participant }>\text { site })=(1-0.44) \cdot 0.5=0.28 .
$$

Consequently, if a participant's system wins more than in $28 \%$ of the impressions, then this is more than expected. And thus the participant's system can be said to be better than the site's system if the outcome is (significantly) more than $28 \%$. Importantly, this applies only to Round \#1. For Round \#2, the probability of winning an interleaving comparison is $50 \%$, therefore an outcome above 0.5 is needed to beat the production system.

\subsection{Submissions}

Two organizations submitted a total of four runs for Round \#1. For Round \#2, three organizations submitted five runs in total. In addition, a simple baseline provided by the challenge organizers is also included for reference. Table 3 presents an overview of participating teams.

Team GESIS [22] downloaded the entire products' metadata set and associated click-through-rate provided by the lab's API. All available metadata was indexed fieldbased into a fresh Solr installation. They applied no language specific configurations for stemmers or stop word lists, as these are not available for Hungarian in the standard configuration of Solr (in fact there is a Hungarian stemmer, but it did not provide different results to the general one). Using the original query strings and ids they obtained a Solr-ranked list of documents for each query. These results were re-ranked using historical click rates. The re-ranking is a linear combination of a boosted search on the document id and the vector space-based relevance score of the query string. This is a typical "the rich are getting richer" approach where formally successful products are 
Table 3. Organizations submitting results to the product search use-case.

\begin{tabular}{|c|c|c|c|c|}
\hline \multicolumn{2}{|c|}{ Team Name Affiliation } & \multicolumn{3}{|c|}{ Location Round \#1 Round \#2 } \\
\hline IRIT [10] & Paul Sabatier University - IRIT & France & - & $\mathrm{Y}$ \\
\hline GESIS [22] & GESIS - Leibniz Institute for the Social Sciences & Germany & $\mathrm{Y}$ & $\mathrm{Y}$ \\
\hline UiS [8] & University of Stavanger & Norway & $\mathrm{Y}$ & $\mathrm{Y}$ \\
\hline
\end{tabular}

more likely to be once again ranked high in the result list. For this, their first attempt using the lab's API they ignored possible ways of improving the retrieval like making use of the underlying categorization system, to filter on available products or to boost special price offers. Many small things that went wrong in Round \#1 that were fixed before the start of Round \#2, hence the large performance difference in the two rounds.

Team IRIT [10] proposed using a probabilistic model for product search based on the intuition that descriptive fields and the category might fit with the query. In their paper, they propose a probabilistic model for product search that addresses the problem of head queries on e-commerce Web sites. Their model relies on product fields, namely the description and the category and ranks products with respect to (i) the likelihood that the product's descriptive fields match the query using the BM25F score, and (ii) the likelihood that the product's category is relevant to the query by analyzing its distribution over the product collection.

Team UiS [8] employed a fielded document retrieval approach based on language modeling techniques. Specifically, building upon the Probabilistic Retrieval Model for Semistructured Data by Kim et al. [15], they experimented with three different methods (UIS-*) for estimating term-field mapping probabilities. Their results show that termspecific field mapping in general is beneficial. They also present evidence suggesting that estimating field mapping priors based on historical clicks outperforms the setting where the priors are uniformly distributed.

The organizers' baseline (BASELINE in Tables 4 and 5) ranks products based on historical click-through rate. Only products that were clicked for the given query are returned; their attributes are not considered. In case historical clicks are unavailable (this happened for a single query R-q97), (all) candidate products are returned in an arbitrary order (in practice, in the same order as they were received from the API via the doclist request, see Section 3.4).

\subsection{Results}

For Round \#1, we find that at least three submissions are likely to have improved upon the production system's ranking. That is, three of the four submissions performed higher than the expected outcome of 0.28, see Section 4.3. Somewhat surprisingly, the simple baseline performed by far the best, with an outcome of 0.4691 . This was also the only system that significantly outperformed the production system. The best performing participant run is UIS-MIRA, with an outcome of 0.3413 .

For Round \#2, the issue with the interleaved comparisons was corrected, see Section 4.3. The expected probability of winning an interleaved comparison (assuming a 
Table 4. Round \#1 results for the product search use-case ordered by Outcome. The expected outcome under a randomly clicking user for is 0.28 , see Section 4.3 . P-values are computed using a binomial test.

\begin{tabular}{lcccccr}
\hline Submission & \multicolumn{7}{c}{ Outcome } & \#Wins & \#Losses & \#Ties \#Impressions & p-value \\
\hline BASELINE & 0.4691 & 91 & 103 & 467 & 661 & $<0.01$ \\
UIS-MIRA [8] & 0.3413 & 71 & 137 & 517 & 725 & 0.053 \\
UIS-JERN [8] & 0.3277 & 58 & 119 & 488 & 665 & 0.156 \\
UIS-UIS [8] & 0.2827 & 54 & 137 & 508 & 699 & 0.936 \\
Expected Outcome & 0.28 & & & & & \\
GESIS [22] & 0.2685 & 40 & 109 & 374 & 523 & 0.785 \\
\hline
\end{tabular}

Table 5. Round \#2 results for the product search use-case ordered by Outcome. The expected outcome under a randomly clicking user for is 0.5 . P-values are computed using a binomial test.

\begin{tabular}{lllcccr}
\hline Submission & \multicolumn{6}{c}{ Outcome } \\
\hline BASELINE & 0.5284 & 93 & 83 & 598 & 774 & 0.498 \\
Expected Outcome & 0.5 & & & & & \\
UIS-JERN [8] & 0.4795 & 82 & 89 & 596 & 767 & 0.646 \\
GESIS [22] & 0.4520 & 80 & 97 & 639 & 816 & 0.229 \\
UIS-MIRA [8] & 0.4389 & 79 & 101 & 577 & 757 & 0.117 \\
UIS-UIS [8] & 0.4118 & 84 & 120 & 527 & 731 & 0.014 \\
IRIT [10] & 0.3990 & 79 & 119 & 593 & 791 & 0.005 \\
\hline
\end{tabular}

randomly clicking user) in this round then was 0.5 . In this round no submission outperformed the production system. However, the baseline, still somewhat surprisingly, performed the best albeit not significantly better than the production system. Given that the corrected outcome in Round \#1 is only an estimate (that might be biased in favor of the submissions), we regard the Round \#2 results a more accurate reflection of system performance.

Apart from the fact that none of the submission systems outperformed the production system in this round, their relative performance also changed. Where UIS-JERN came second in Round \#1, it won in Round \#2, which is more in line with the expectation of the team that submitted this system and the one it overtook. Even more strikingly is the large jump in relative system performance for GESIS. This can most likely be explained by numerous fixes to problems encountered in Round \#1. Other than this it is hard to draw any conclusions from these differences as almost all of them have extremely high p-values. We would require more data in order to obtain more stable results before we can draw any conclusions. 


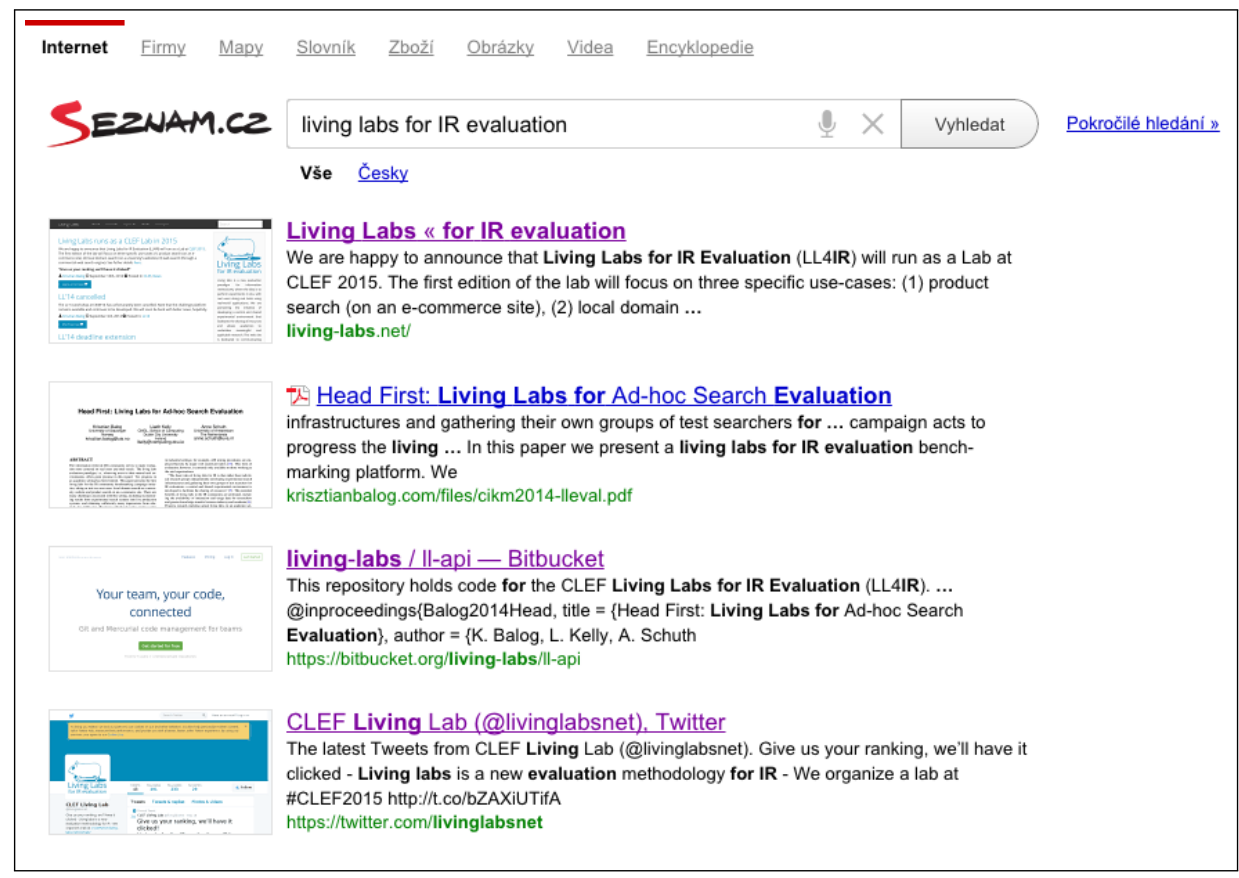

Fig. 5. Screenshot of Seznam, our web search use-case.

\section{Use-case 2: Web Search}

\subsection{Task and Data}

The web search use-case is provided by Seznam, ${ }^{11}$ a very large web search engine in the Czech Republic. See Figure 5 for a screenshot of the user interface.

Seznam serves almost half the country's search traffic and as such has very high site traffic. Queries are the typical web search queries, and thus are a mixed bag of transactional, navigational and transactional [5]. In contrast to the product search usecase, apart from the scale and the query types, Seznam does not make raw document and query content available, rather features computed for documents and queries. This is much like any learning to rank dataset, such as Letor [18]. Queries and documents are only identified by a unique identifier and for each query, the candidate documents are represented with sparse feature vectors. Seznam provided a total of 557 features. These features were not described in any way. The challenge with this use-case then is a learning to rank challenge [17].

As described in Section 3, the web search use-case also consists of a training and test phase. For the test phase, there were 97 queries. For the training phase 100 queries were provided. On average, for each query there were about 179 candidate documents. In total, there were 35,322 documents.

${ }^{11}$ http://search.seznam.cz 


\subsection{Implementation}

Every time one of the selected queries is issued by a user for the first time, Seznam makes a call to our API while simultaneously calling the production rankers. When our API responds too slow $(>100 \mathrm{~ms})$ it is ignored completely and the production ranking is shown. When our API does respond in time, the participants' ranking is interleaved with the production ranking. Seznam implemented Team Draft Interleaving (TDI) [20] themselves. Users are always provided with stable rankings, so if a user issues the same query twice, a cached results is shown. Seznam implements this caching mechanism.

\subsection{Results}

The web search use-case attracted 6 teams that submitted runs for the training queries. However, none of these teams submitted runs for the test queries. Therefore, we can only report on two baseline systems, provided by the challenge organizers. Baseline 1, titled Exploitative Baseline in Tables 6 and 7, uses the original Seznam ranking and was therefore expected to produce an outcome of 0.5. ${ }^{12}$ Baseline 2, titled UNIFORM BASELINE in Tables 6 and 7, assigned uniform weights to each feature and ranked by the weighted sum of feature values. This baseline was expected to not perform well.

Over the past months, there have been over 480K impressions on Seznam through our Living Labs API. On average this amounts to 2,428 impressions for each query. Approximately $6 \%$ of all impressions were used for the testing period. As can be seen in Table 6, the EXPLOITATIVE BASELINE outperformed the production system. An outcome (outcome measure described in Section 3) of 0.5527 was achieved, with 3,030 wins and 2,452 losses against the production system, and 19,055 ties with it. As expected, the UNIFORM BASELINE lost many more comparisons than it won. Both outcomes were statistically significant according to a binomial test.

Results for Round \#2 are qualitatively the same as for Round \#1. There were approximately the same number of impressions during this period and again one baseline is better and one worse than the expected outcome. Both significantly so. Both baselines did improve upon themselves though. This is a rather surprising result as the baselines did not update their rankings after Round \#1 and therefore it is likely that some documents whet stale while other new documents were not included at all. This should have resulted in reduced performance. We do not yet have a good explanation for why this may be the case.

\section{Discussion and Conclusions}

The living labs methodology offers great potential to evaluate information retrieval systems in live settings with real users. The Living Labs for Information Retrieval Evaluation (LL4IR) CLEF Lab represents the first attempt at a shared community benchmarking platform in this space. The first edition of LL4IR focused on two use-cases, product

\footnotetext{
${ }^{12}$ If use-cases uploaded their candidate documents in the order that represented their own ranking, then this was available to participants. We plan to change this in the future.
} 
Table 6. Round \#1 results for the web search use-case. The expected outcome under a randomly clicking user is 0.5 . P-values were computed using a binomial test.

\begin{tabular}{|c|c|c|c|c|c|c|}
\hline Submission & Outcome & \#Wins & \#Losses & \#Ties & \#Impressic & p-value \\
\hline EXPLOITATIVE BASELINE & 0.5527 & 3030 & 2452 & 19055 & 24537 & $<0.01$ \\
\hline Expected Outcome & 0.5 & & & & & \\
\hline UNIFORM BASELINE & 0.2161 & 430 & 1560 & 1346 & 3336 & $<0.01$ \\
\hline
\end{tabular}

Table 7. Round \#2 results for the web search use-case. The expected outcome under a randomly clicking user is 0.5 . P-values were computed using a binomial test.

\begin{tabular}{lllllll}
\hline Submission & \multicolumn{6}{c}{ Outcome } \\
\hline EXPLOITATIVE BASELINE & 0.6035 & 3128 & 2055 & 18055 & 23238 & $<0.01$ \\
Expected Outcome & 0.5 & & & & & \\
UNIFORM BASELINE & 0.2547 & 435 & 1273 & 1053 & 2761 & $<0.01$ \\
\hline
\end{tabular}

search and web search, using a commercial e-commerce website, REGIO, and a commercial web search engine, Seznam. A major contribution of the lab is the development of the necessary API infrastructure, which is made publicly available.

The LL4IR CLEF Lab attracted interest from dozens of teams. There were 12 active participants, but only 3 teams ended up submitting results for the official evaluation (excluding the baseline systems, provided by the organizers). We found that, while many researchers expressed and showed their interest in the lab, our setup with an API, instead of a static test collection, was a hurdle for many. We plan to ease this process of adapting to this new evaluation paradigm by providing even more examples and by organizing tutorials where we demonstrate working with our API.

Overall, we regard our effort successful in showing the feasibility and potential of this form of evaluation. For both use-cases, there was an experimental system that outperformed the corresponding production system significantly. It is somewhat unfortunate that in both cases that experimental system was a baseline approach provided by the challenge organizers, nevertheless, it demonstrates the potential benefits to use-case owners as well. One particular issue that surfaced and needs addressing for the product search use-case is the frequent changes in inventory. This appears to be more severe than we first anticipated and represents some challenges, both technical and methodological.

The API infrastructure developed for the LL4IR CLEF Lab offers the potential to host ongoing IR evaluations in a live setting. As such, it is planned that these "challenges" will continue on an ongoing basis post-CLEF, with an expanding number of use-cases as well as refinements to the existing use-cases. ${ }^{13}$ In fact, a third round of our evaluation challenge is already underway at the time of writing, with some modifications to the initial setup. Moreover, this third round has already attracted more participants, in particular for the web search use case.

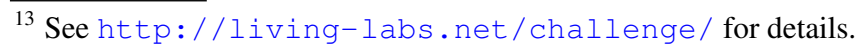




\section{Acknowledgements}

We would like to acknowledge the support of (in alphabetical order): the CLEF Initiative; the Dutch national program COMMIT; the ESF ELIAS network program; the REGIO Játék online toy store; and Seznam commercial search engine. We would also like to thank the participants for their submissions and interest in the lab.

\section{Bibliography}

[1] J. Allan, B. Croft, A. Moffat, and M. Sanderson. Frontiers, challenges, and opportunities for information retrieval: Report from SWIRL 2012 the second strategic workshop on information retrieval in lorne. SIGIR Forum, 46(1):2-32, 2012.

[2] L. Azzopardi and K. Balog. Towards a living lab for information retrieval research and development. A proposal for a living lab for product search tasks. In CLEF' 11, 2011.

[3] K. Balog, D. Elsweiler, E. Kanoulas, L. Kelly, and M. D. Smucker. Report on the CIKM workshop on living labs for information retrieval evaluation. SIGIR Forum, 48(1):21-28, 2014.

[4] K. Balog, L. Kelly, and A. Schuth. Head first: Living labs for ad-hoc search evaluation. In CIKM'14, 2014.

[5] A. Broder. A taxonomy of web search. SIGIR Forum, 36(2):3-10, Sept. 2002. ISSN 01635840.

[6] T. Brodt and F. Hopfgartner. Shedding light on a living lab: The CLEF NEWSREEL open recommendation platform. In IIiX'14, 2014.

[7] O. Chapelle, T. Joachims, F. Radlinski, and Y. Yue. Large-scale validation and analysis of interleaved search evaluation. ACM Transactions on Information Systems (TOIS), 30:1-41, 2012.

[8] A. B. Ghirmatsion and K. Balog. Probabilistic field mapping for product search. In CLEF 2015 Online Working Notes, 2015.

[9] K. Hofmann, S. Whiteson, and M. de Rijke. A probabilistic method for inferring preferences from clicks. In CIKM'11, page 249, 2011.

[10] L. B. Jabeur, L. Soulier, and L. Tamine. IRIT at CLEF 2015: A product search model for head queries. In CLEF 2015 Online Working Notes, 2015.

[11] T. Joachims. Evaluating retrieval performance using clickthrough data. In J. Franke, G. Nakhaeizadeh, and I. Renz, editors, Text Mining, pages 79-96. Physica/Springer, 2003.

[12] J. Kamps, S. Geva, C. Peters, T. Sakai, A. Trotman, and E. Voorhees. Report on the SIGIR 2009 workshop on the future of IR evaluation. SIGIR Forum, 43(2): 13-23, 2009.

[13] D. Kelly, S. Dumais, and J. O. Pedersen. Evaluation challenges and directions for information-seeking support systems. Computer, 42(3):60-66, 2009.

[14] L. Kelly, P. Bunbury, and G. J. F. Jones. Evaluating personal information retrieval. In ECIR'12, 2012.

[15] J. Kim, X. Xue, and W. B. Croft. A probabilistic retrieval model for semistructured data. In ECIR'09, 2009. 
[16] R. Kohavi. Online controlled experiments. In SIGIR'13, 2013.

[17] T.-Y. Liu. Learning to rank for information retrieval. Found. Trends Inf. Retr., 3 (3):225-331, 2009.

[18] T.-Y. Liu, J. Xu, T. Qin, W. Xiong, and H. Li. LETOR: Benchmark dataset for research on learning to rank for information retrieval. In $L R 4 I R^{\prime} 07,2007$.

[19] F. Radlinski and N. Craswell. Optimized interleaving for online retrieval evaluation. In WSDM'13, 2013.

[20] F. Radlinski, M. Kurup, and T. Joachims. How does clickthrough data reflect retrieval quality? In CIKM'08, 2008.

[21] M. Sanderson. Test Collection Based Evaluation of Information Retrieval Systems. Foundations and Trends in Information Retrieval, 4(4):247-375, 2010.

[22] P. Schaer and N. Tavakolpoursaleh. GESIS at CLEF LL4IR 2015. In CLEF 2015 Online Working Notes, 2015.

[23] A. Schuth, F. Sietsma, S. Whiteson, D. Lefortier, and M. de Rijke. Multileaved comparisons for fast online evaluation. In CIKM'14, 2014.

[24] A. Schuth, R.-J. Bruintjes, F. Büttner, J. van Doorn, C. Groenland, H. Oosterhuis, C.-N. Tran, B. Veeling, J. van der Velde, R. Wechsler, D. Woudenberg, and M. de Rijke. Probabilistic multileave for online retrieval evaluation. In SIGIR'15, 2015.

[25] A. Schuth, K. Hofmann, and F. Radlinski. Predicting search satisfaction metrics with interleaved comparisons. In SIGIR'15, 2015.

[26] A. Spink, D. Wolfram, M. B. J. Jansen, and T. Saracevic. Searching the web: The public and their queries. J. Am. Soc. Inf. Sci. Technol., 52(3):226-234, 2001.

[27] J. Teevan, S. Dumais, and E. Horvitz. The potential value of personalizing search. In SIGIR'07, pages 756-757, 2007.

[28] A. Turpin and F. Scholar. User performance versus precision measures for simple search tasks. In SIGIR'06, pages 11-18, 2006. 